\title{
Spinning Particle with Anomalous Magnetic Moment in an External Plane Wave Field.
}

\author{
A. Barducci and R.Giachetti \\ Department of Physics, University of Florence and I.N.F.N. Sezione di Firenze \\ Via G. Sansone 1, I-50019 Sesto Fiorentino, Firenze, Italy1
}

Firenze Preprint - DFF - 445/12/07

\begin{abstract}
In this paper we study the interaction of a Dirac-Pauli particle with an electromagnetic plane wave, by using a previously given generalization of the pseudo-classical Lagrangian for a spinning particle with an anomalous magnetic moment. We derive the explicit expressions for the eigenfunctions and the Green's functions of the theory. We discuss the validity of the semi-classical approach by comparing the wavefunctions with the (pseudo)-classical solutions of the Hamilton-Jacobi equation.
\end{abstract}

\section{Introduction.}

Starting with the papers [1, 3], the description of relativistic spinning particles and superparticles has found many other different formulations in past years [4]-[10]. The study of these models was firstly originated by their close relation with the string theory, but it later became clear that the problem was important in itself for a deeper understanding of the structure of the quantum theory. These models have been later investigated and quantized also in the presence of external fields both by means of canonical quantization and by the use of the path- integral. More refined results have been achieved when the external fields were taken as plane waves: in these cases it has been proved, either by path integral [7, 11, 12] and by canonical theory [13, 14], that the complete quantum propagator coincides with the results of the semi-classical approximation.

\footnotetext{
${ }^{1}$ e-address: barducci@fi.infn.it, giachetti@fi.infn.it
} 
The description of a spinning particle with anomalous magnetic moment has firstly been given in [15] and later on, with different approaches, in [16, 17, 18]. All of these treatments lead to the same first class Dirac constraints and therefore to the same quantum wave equation.

The purpose of this work is to study the quantization of a pseudo-classical spinning particle which has both the normal magnetic moment and an anomalous magnetic moment in the field of a plane electromagnetic wave, thus completing the research begun in [14] for the usual spinning particle. As we shall show, the main difference with respect to the previous cases is that now the semi-classical approximation is no longer exact except for some particular cases: this is due to the interference of the anomalous magnetic moment with the electric charge, which makes the presence of a $T$-ordered product in the propagator unavoidable at the quantum level.

We begin in Section 2 with the description of the model for the spinning particle with anomalous magnetic moment and we derive the two constraints of the theory and we study the corresponding canonical quantization procedure. One of these constraints - the Diraclike one - is linear; the other - the Klein-Gordon-like - is quadratic. Obviously the latter involves some ordering difficulties in the quantization procedure. However, since the Poisson bracket of the linear constraint gives rise to the quadratic constraint, we will quantize the system by following the Dirac procedure based on the correspondence principle. We recall that this procedure is based on the postulate that the Poisson brackets for the the quantum mechanical variables (which are operators in a Hilbert space) must satisfy the same algebraic identities as the classical ones; it then follows that the Poisson brackets are proportional to the (anti)-commutator. We will therefore assume this Dirac prescription for quantizing the theory. It is interesting to notice that the expression we find for the quantized quadratic constraint, following this procedure, is the same we would find by quantizing the theory with the standard Weyl symmetrization of the quantum dynamical variables. This therefore makes every definition well posed. In Section 3. we calculate the wavefunction for the DiracPauli particle and we derive the Green's function of the theory. Finally, in Section 4, we make a comparison with the semi-classical approximation that can be obtained by solving the corresponding pseudo-classical Hamilton-Jacobi equation.

\section{Pseudoclassical description and canonical quantiza- tion.}

As we said in the introduction, there are different approaches to the description of a pseudoclassical spinning particle both in the standard case and in the case of a particle with anomalous magnetic moment. Since all of them lead to the same constraints and therefore to the same quantum wave equation, we start with the Lagrangian given in [15], namely 


$$
\begin{aligned}
& \mathrm{L}\left(x_{\mu}, \dot{x}_{\mu}, \xi_{\mu}, \dot{\xi}_{\mu}, \xi_{5}, \dot{\xi}_{5}\right)= \\
& -\frac{i}{2}(\xi \cdot \dot{\xi})-\frac{i}{2} \xi_{5} \dot{\xi}_{5}-q(\dot{x} \cdot A)-\left[m^{2}-i\left(q+\frac{e \mu}{2}\right) F_{\mu \nu} \xi^{\mu} \xi^{\nu}-\frac{e^{2} \mu^{2}}{16 m^{2}} F_{\mu \nu} F_{\rho \sigma} \xi^{\mu} \xi^{\nu} \xi^{\rho} \xi^{\sigma}\right]^{1 / 2} \\
& \quad\left[\left(\dot{x}^{\mu}-i\left(m+\frac{i e \mu}{4 m} F_{\mu \nu} \xi^{\mu} \xi^{\nu}\right)^{-1} \xi^{\mu}\left(\dot{\xi}_{5}-\frac{e \mu}{2 m} \dot{x}_{\rho} F_{\rho \sigma} \xi^{\sigma}\right)\right)^{2}\right]^{1 / 2}
\end{aligned}
$$

We use here the standard convention for the metric tensor and for the gamma matrices [19]. Here $q$ means the charge of the particle and $e$ the electronic charge respectively. After using the second class constraints arising from the Lagrangian, the final form for the two first class constraints becomes

$$
\begin{gathered}
\chi_{D}=((p-q A) \cdot \xi)-m \xi_{5}+i \frac{e \mu}{4 m} F_{\mu \nu} \xi^{\mu} \xi^{\nu} \xi_{5} \\
\chi=(p-q A)^{2}-m^{2}+i\left(q+\frac{e \mu}{2}\right) F_{\mu \nu} \xi^{\mu} \xi^{\nu}+i \frac{e \mu}{m}\left(p^{\mu}-q A^{\mu}\right) F_{\mu \nu} \xi^{\nu} \xi_{5}+ \\
\frac{e^{2} \mu^{2}}{16 m^{2}} F_{\mu \nu} F_{\rho \sigma} \xi^{\mu} \xi^{\nu} \xi^{\rho} \xi^{\sigma}
\end{gathered}
$$

The relevant Dirac brackets for the pseudo-classical variables are

$$
\left\{x^{\mu}, p^{\nu}\right\}=-\eta^{\mu \nu} \quad\left\{\xi^{\mu}, \xi^{\nu}\right\}=i \eta^{\mu \nu}, \quad\left\{\xi_{5}, \xi_{5}\right\}=-i
$$

leading to the constraint's algebra

$$
\left\{\chi_{D}, \chi_{D}\right\}=i \chi, \quad\left\{\chi_{D}, \chi\right\}=\{\chi, \chi\}=0
$$

Because the algebra of the pseudo-classical Poisson brackets is a graded Lie algebra it follows that the quantum Poisson brackets must be proportional to an anticommutator for the FermiFermi case and to a commutator for the Bose-Bose and the mixed Bose-Fermi cases. The graded quantum commutation rules for the canonical variables read therefore

$$
\left[x^{\mu}, p^{\nu}\right]=-i \eta^{\mu \nu}, \quad\left\{\hat{\xi}^{\mu}, \hat{\xi}^{\nu}\right\}_{+}=-\eta^{\mu \nu}, \quad\left\{\hat{\xi}_{5}, \hat{\xi}_{5}\right\}_{+}=1
$$

In order to satisfy the anti-commutation relation for the $\hat{\xi}$ variables, we see that a possible realization can be the following:

$$
\hat{\xi}^{\mu}=2^{-1 / 2} \gamma_{5} \gamma^{\mu}, \quad \hat{\xi}_{5}=2^{-1 / 2} \gamma_{5}
$$

The explicit form of the quantized Dirac linear constraint takes now the form

$$
\hat{\chi}_{D}=2^{-1 / 2} \gamma_{5}\left((\gamma \cdot(p-q A))-\frac{e \mu}{8 m} \sigma_{\mu \nu} F^{\mu \nu}-m\right) \equiv 2^{-1 / 2} \gamma_{5} \hat{\mathcal{O}}_{D}
$$

where $\sigma^{\mu \nu}$ is defined by $\sigma^{\mu \nu}=(i / 2)\left[\gamma^{\mu}, \gamma^{\nu}\right]$. 
As we said in the introduction, the quantization of the quadratic constraint involves some ordering problems, since the constraint $\chi$ contains terms with product of the momenta and functions of the coordinates. A correct way of solving this ambiguity is to define (following the Dirac correspondence principle procedure) the quantum expression of this constraint according to the quantized version of equation (5), so that

$$
\hat{\chi}=-2 \hat{\chi}_{D}^{2}=-\frac{1}{2}\left\{\gamma_{5} \hat{\mathcal{O}}_{D}, \gamma_{5} \hat{\mathcal{O}}_{D}\right\}_{+}
$$

and explicitly

$$
\begin{gathered}
\hat{\chi}=(p-q A)^{2}-\frac{1}{2}\left(q+\frac{e \mu}{2}\right) \sigma_{\mu \nu} F^{\mu \nu}-i \frac{e \mu}{8 m} \sigma^{\mu \nu} \gamma^{\rho} \partial_{\rho} F_{\mu \nu}-\frac{e \mu}{2 m} \gamma^{\mu} \partial^{\nu} F_{\mu \nu}+ \\
i \frac{e \mu}{2 m} \gamma^{\mu} F_{\mu \nu}\left(p^{\nu}-q A^{\nu}\right)-\left(\frac{e \mu}{8 m}\right)^{2} \sigma_{\mu \nu} \sigma_{\rho \sigma} F^{\mu \nu} F^{\rho \sigma}-m^{2}
\end{gathered}
$$

By now using the relation $\sigma^{\mu \nu} \gamma^{\rho} \partial_{\rho} F_{\mu \nu}=(i / 2)\left[\gamma^{\mu}, \gamma^{\nu}\right] \gamma^{\rho} \partial_{\rho} F_{\mu \nu}=2 i \gamma^{\mu} \partial^{\nu} F_{\mu \nu}$, (we recall $\partial^{\nu} F_{\mu \nu}^{*}=$ 0 , where $F_{\mu \nu}^{*}$ is the dual electromagnetic tensor) we will get for the final form of the quantized quadratic constraint

$$
\begin{aligned}
\hat{\chi}=(p-q A)^{2}- & \frac{1}{2}\left(q+\frac{e \mu}{2}\right) F^{\mu \nu} \sigma_{\mu \nu}-\frac{e \mu}{4 m} \gamma^{\mu} \partial^{\nu} F_{\mu \nu}+ \\
& i \frac{e \mu}{2 m} \gamma^{\mu} F_{\mu \nu}\left(p^{\nu}-q A^{\nu}\right)-\left(\frac{e \mu}{8 m}\right)^{2} F^{\mu \nu} F^{\rho \sigma} \sigma_{\mu \nu} \sigma_{\rho \sigma}-m^{2}
\end{aligned}
$$

The commonly adopted approach for solving the previous ordering ambiguities is to use the Weyl symmetrization prescription for the classical expressions, which in particular provides the hermiticity of the corresponding quantum operators. Although in principle this procedure does not guarantee that the relevant algebraic structures are maintained at the quantum level, in the present case (as we said in the introduction) the Weyl symmetrization procedure would lead to the following quantized version of the constraint:

$$
\begin{aligned}
\hat{\chi}_{\mathrm{Weyl}}= & (p-q A)^{2}+i \frac{e \mu}{2 m}\left\{p^{\mu}-q A^{\mu}, F_{\mu \nu}\right\}_{+} \hat{\xi}^{\nu} \xi_{5}+i\left(q+\frac{e \mu}{2}\right) F^{\mu \nu} \hat{\xi}_{\mu} \hat{\xi}_{\nu}+ \\
& \left(\frac{e \mu}{4 m}\right)^{2} F^{\mu \nu} F^{\rho \sigma} \hat{\xi}_{\mu} \hat{\xi}_{\nu} \hat{\xi}_{\rho} \hat{\xi}_{\sigma}-m^{2}
\end{aligned}
$$

By using the identity

$$
\begin{aligned}
\frac{1}{2}\left\{p^{\mu}-q A^{\mu}, F_{\mu \nu}\right\}_{+} \hat{\xi}^{\nu} \xi_{5} & =-\frac{1}{4}\left\{p_{\mu}-q A_{\mu}, F_{\mu \nu}\right\}_{+} \gamma^{\nu}=\frac{i}{8}\left[(\gamma \cdot(p-q A)), F^{\mu \nu} \sigma_{\mu \nu}\right] \\
& =\frac{i}{4} \gamma^{\mu}\left(\partial^{\nu} F_{\mu \nu}\right)+\frac{1}{2} \gamma^{\mu} F_{\mu \nu}\left(p^{\nu}-q A^{\nu}\right)
\end{aligned}
$$

it is easily seen that the expression (12) coincides with (11). Therefore we have shown that the quadratic wave equation appears to be a mere consequence of the first order wave equation, i.e. the Dirac-Pauli equation.

Finally the equation obtained by squaring the linear Dirac operator is therefore the following:

$$
\left[(p-q A)^{2}-\frac{1}{2}\left(q+\frac{e \mu}{2}\right) F^{\mu \nu} \sigma_{\mu \nu}-\frac{e \mu}{4 m} \gamma^{\mu} \partial^{\nu} F_{\mu \nu}+\right.
$$




$$
\left.i \frac{e \mu}{2 m} \gamma^{\mu} F_{\mu \nu}\left(p^{\nu}-q A^{\nu}\right)-\left(\frac{e \mu}{8 m}\right)^{2} F^{\mu \nu} F^{\rho \sigma} \sigma_{\mu \nu} \sigma_{\rho \sigma}-m^{2}\right] \psi=0
$$

We now assume that the electromagnetic potential $A^{\mu}$ describes the field of an external plane wave and has thus the following dependence upon the space-time variables

$$
A^{\mu}=a^{\mu} f(\phi)
$$

where $\phi=(k \cdot x)$ with $k^{2}=0$ and where we assume the Lorentz gauge, so that also $(k \cdot a)=0$. From this potential we get the electromagnetic tensor $\left(f^{\prime}(\phi)=d f(\phi) / d \phi\right)$

$$
F^{\mu \nu}=f^{\mu \nu} f^{\prime}(\phi), \quad f^{\mu \nu}=k^{\mu} a^{\nu}-k^{\nu} a^{\mu}
$$

and the final expression for the wave equation to be solved reads

$$
\begin{aligned}
& {\left[\partial^{2}+2 i q(A \cdot \partial)-q^{2} A^{2}+i\left(q+\frac{e \mu}{2}\right)(\gamma \cdot k)\left(\gamma \cdot A^{\prime}\right)\right.} \\
& \left.\quad-i \frac{e \mu}{2 m}\left(i(\gamma \cdot k)\left(A^{\prime} \cdot \partial\right)-q(\gamma \cdot k)\left(A^{\prime} \cdot A\right)-i\left(\gamma \cdot A^{\prime}\right)(k \cdot \partial)\right)+m^{2}\right] \psi(x)=0
\end{aligned}
$$

\section{The wave function and the Green's function}

Recalling the standard derivation of the Volkov solutions for the usual Dirac and Klein-Gordon equations [13, 20], we make for the wave function the ansatz

$$
\psi(x)=\frac{1}{\left(2 p_{0} V\right)^{1 / 2}} \exp \left\{-i(p \cdot x)-\frac{i}{2(k \cdot p)} \int_{-\infty}^{(k \cdot x)} d \phi\left[2 q(A \cdot p)-q^{2} A^{2}\right]\right\} M((k \cdot x)) u(p, s)
$$

where $M((k \cdot x))$ is a $4 \times 4$ unknown Dirac matrix and where the initial condition

$$
\psi(x) \underset{x_{0} \rightarrow-\infty}{\longrightarrow}\left(2 p_{0} V\right)^{-1 / 2} \exp [-i(p \cdot x)] u(p, s)
$$

is assumed. In equation (19) $u(p, s)$ is a constant spinor which is is a solution of the corresponding free Dirac equation. Moreover, since the redefinition of $p^{\mu}$ as $p^{\mu}+\alpha k^{\mu}$ leaves invariant the equation (18) due to the fact that $M((k \cdot x))$ is a unknown function to be determined, we can impose on the constant vector $p^{\mu}$ the mass-shell relation $p^{2}=m^{2}$. The differential equation satisfied by $M$ is therefore

$$
\begin{aligned}
\frac{d M}{d \phi}=\frac{1}{2(k \cdot p)}[(q & \left.+\frac{e \mu}{2}\right)(\gamma \cdot k)\left(\gamma \cdot A^{\prime}\right) \\
& \left.-\frac{e \mu}{2 m}\left(\left(A^{\prime} \cdot p\right)(\gamma \cdot k)-q\left(A^{\prime} \cdot A\right)(\gamma \cdot k)-(k \cdot p)\left(\gamma \cdot A^{\prime}\right)\right)\right] M(\phi)
\end{aligned}
$$

and the formal solution of the previous equation is

$$
M((k \cdot x))=T\left\{\operatorname { e x p } \left[\frac { 1 } { 2 ( k \cdot p ) } \int _ { - \infty } ^ { ( k \cdot x ) } d \phi \left(\left(q+\frac{e \mu}{2}\right)(\gamma \cdot k)\left(\gamma \cdot A^{\prime}\right)-\right.\right.\right.
$$




$$
\left.\left.\left.\frac{e \mu}{2 m}\left(\left(A^{\prime} \cdot p\right)(\gamma \cdot k)-q\left(A^{\prime} \cdot A\right)(\gamma \cdot k)-(k \cdot p)\left(\gamma \cdot A^{\prime}\right)\right)\right)\right]\right\}
$$

with the asymptotic condition $M(-\infty)=\mathbf{1}$. The final expression for the wave equation reads

$$
\begin{array}{r}
\psi(x)=\frac{1}{\left(2 p_{0} V\right)^{1 / 2}} \exp \left[-i(p \cdot x)-\frac{i}{2(k \cdot p)} \int_{-\infty}^{k \cdot x} d \phi\left(2 q(A \cdot p)-q^{2} A^{2}\right)\right] \\
T\left\{\operatorname { e x p } \left[\frac { 1 } { 2 ( k \cdot p ) } \int _ { - \infty } ^ { ( k \cdot x ) } d \phi \left(\left(q+\frac{e \mu}{2}\right)(\gamma \cdot k)\left(\gamma \cdot A^{\prime}\right)-\frac{e \mu}{2 m}\left(\left(A^{\prime} \cdot p\right)(\gamma \cdot k)-\right.\right.\right.\right. \\
\left.\left.\left.\left.q\left(A^{\prime} \cdot A\right)(\gamma \cdot k)-(k \cdot p)\left(\gamma \cdot A^{\prime}\right)\right)\right)\right]\right\} u(p, s)
\end{array}
$$

The expression (22) for $\psi(x)$ is obviously a formal one. However there are at least two special cases in which from (22) we can get a closed form for this solution. The first of these cases occurs when $q \neq 0$ and $\mu=0$, that is the special case of no anomalous magnetic moment but a charged particle. This choice leads immediately to the Volkov solution

$$
\psi(x)=\frac{1}{\left(2 p_{0} V\right)^{1 / 2}} \exp \left\{-i(p \cdot x)-\frac{i}{2(k \cdot p)} \int_{-\infty}^{(k \cdot x)} d \phi\left(2 q(A \cdot p)-q^{2} A^{2}+\frac{q}{2} F^{\mu \nu} \sigma_{\mu \nu}\right)\right\} u(p, s)
$$

The second case, with $q=0$ and $\mu \neq 0$, i.e. the special case of pure anomalous magnetic moment and no charge, gives a new result in a closed form. Indeed we obtain

$$
\begin{array}{r}
\psi(x)=\frac{1}{\left(2 p_{0} V\right)^{1 / 2}} \exp \left\{-i(p \cdot x)+\frac{e \mu}{4(k \cdot p)} \int_{-\infty}^{(k \cdot x)} d \phi\left[(\gamma \cdot k)\left(\gamma \cdot A^{\prime}\right)-\right.\right. \\
\left.\left.\frac{1}{m}\left(\left(A^{\prime} \cdot p\right)(\gamma \cdot k)-(k \cdot p)\left(\gamma \cdot A^{\prime}\right)\right)\right]\right\} u(p, s)
\end{array}
$$

It can be realized that the presence of the time ordering is due to the joint action of the terms involving the anomalous magnetic moment $\mu$ and the electric charge $q$. When only one of these components is effective, the time ordering can be calculated and an expression in closed form is obtained.

Similar procedures to the ones we have used for calculating the wavefunction, can be used also for the computation of the Green's function $S_{F}(x, y)$ that satisfies

$$
\hat{\mathcal{O}}_{D} S_{F}(x, y)=\delta^{4}(x-y)
$$

where $\hat{\mathcal{O}}_{D}$ is given in (8). As usual, instead of solving directly the first-order Dirac equation (25), it is easier to consider the corresponding second order equation

$$
\hat{\chi} \Delta_{F}(x, y)=\delta^{4}(x-y)
$$


where $\Delta_{F}(x, y)$ is defined by (see eq.(91))

$$
\gamma_{5} S_{F}(x, y) \gamma_{5}=-\hat{\mathcal{O}}_{D} \Delta_{F}(x, y)
$$

The general second-order wave equation for the Green's function is therefore

$$
\begin{aligned}
& {\left[\partial^{2}+2 i q(A \cdot \partial)-q^{2} A^{2}+\frac{1}{2}\left(q+\frac{e \mu}{2}\right) F^{\mu \nu} \sigma_{\mu \nu}+\frac{e \mu}{4 m} \gamma^{\mu} \partial^{\nu} F_{\mu \nu}-\right.} \\
& \left.\quad \frac{i \mu e}{2 m} \gamma^{\mu} F_{\mu \nu}\left(i \partial^{\nu}-q A^{\nu}\right)+\left(\frac{e \mu}{8 m}\right)^{2} F^{\mu \nu} F^{\rho \tau} \sigma_{\mu \nu} \sigma_{\rho \tau}+m^{2}\right] \Delta_{F}(x, y)=-\delta^{4}(x-y)
\end{aligned}
$$

and, by choosing $A^{\mu}$ as in (15) we obtain,

$$
\begin{aligned}
& {\left[\partial^{2}+2 i q(A \cdot \partial)-q^{2} A^{2}+i\left(q+\frac{e \mu}{2}\right)(\gamma \cdot k)\left(\gamma \cdot A^{\prime}\right)-\right.} \\
& \left.\quad i \frac{e \mu}{2 m}\left(i(\gamma \cdot k)\left(A^{\prime} \cdot \partial\right)-i\left(\gamma \cdot A^{\prime}\right)(k \cdot \partial)-q(\gamma \cdot k)\left(A^{\prime} \cdot A\right)\right)+m^{2}\right] \Delta_{F}(x, y)=-\delta^{4}(x-y)
\end{aligned}
$$

To solve (29) we assume as ansatz

$$
\Delta_{F}(x, y)=\int \frac{d^{4} p}{(2 \pi)^{4}} \frac{\exp \{-i(p \cdot(x-y))\}}{p^{2}-m^{2}} \exp \left\{-i F_{1}\left(\phi_{x}, \phi_{y}\right)\right\} N\left(\phi_{x}, \phi_{y}\right)
$$

where $\phi_{x}=(k \cdot x), \phi_{y}=(k \cdot y)$ and

$$
F_{1}\left(\phi_{x}, \phi_{y}\right)=\frac{1}{2(k \cdot p)} \int_{(k \cdot y)}^{(k \cdot x)} d \phi\left(2 q(A \cdot p)-q^{2} A^{2}\right)
$$

By inserting the ansatz (30) into (29) we obtain the following equation for $N\left(\phi_{x}, \phi_{y}\right)$ :

$$
\begin{gathered}
\int \frac{d^{4} p}{(2 \pi)^{4}} \exp \{-i(p \cdot(x-y))\}\left[1-\exp \left\{-i F_{1}\left(\phi_{x}, \phi_{y}\right)\right\} N\left(\phi_{x}, \phi_{y}\right)\right]= \\
\int \frac{d^{4} p}{(2 \pi)^{4}} \exp \left\{-i(p \cdot(x-y))-i F_{1}\left(\phi_{x}, \phi_{y}\right)\right\}\left(\frac{-2 i(k \cdot p)}{p^{2}-m^{2}}\right)\left[\frac{d N}{d \phi_{x}}-\frac{1}{2(k \cdot p)}\left(\left(q+\frac{e \mu}{2}\right)(\gamma \cdot k)\left(\gamma \cdot A^{\prime}\right)-\right.\right. \\
\left.\left.\frac{e \mu}{2 m}\left((\gamma \cdot k)\left(A^{\prime} \cdot p\right)-q(\gamma \cdot k)\left(A^{\prime} \cdot A\right)-\left(\gamma \cdot A^{\prime}\right)(k \cdot p)\right)\right) N\left(\phi_{x}, \phi_{y}\right)\right]
\end{gathered}
$$

We can now observe that the expression in the square bracket in the right hand side of equation (32) coincides with (20), up to the obvious substitutions. The right hand side of (32) is therefore vanishing by assuming for $N\left(\phi_{x}, \phi_{y}\right)$ the form

$$
\begin{aligned}
N\left(\phi_{x}, \phi_{y}\right)=T\left\{\operatorname { e x p } \left[\frac{1}{2(k \cdot p)} \int_{\phi_{y}}^{\phi_{x}}\left(q+\frac{e \mu}{2}\right)(\gamma \cdot k)\left(\gamma \cdot A^{\prime}\right)-\right.\right. \\
\left.\left.\frac{e \mu}{2 m}\left((\gamma \cdot k)\left(A^{\prime} \cdot p\right)-q(\gamma \cdot k)\left(A^{\prime} \cdot A\right)-\left(\gamma \cdot A^{\prime}\right)(k \cdot p)\right)\right]\right\}
\end{aligned}
$$


We have therefore to verify that the left hand side of (32) is also vanishing. We first separate in the left hand side of (32) the component of $p_{\mu}$ in the direction of $k_{\mu}, p^{\mu}=p^{\prime^{\mu}}+\alpha k^{\mu}$ where $p^{\prime \mu}$ spans a three-dimensional surface, [20]. Since the function $F_{1}\left(\phi_{x}, \phi_{y}\right)$ is independent of $\alpha$ we can integrate over $\alpha$, getting

$$
\begin{aligned}
\int \frac{d \alpha}{2 \pi} \exp \left[-i \alpha\left(\phi_{x}-\phi_{y}\right)\right] & \left(1-\exp \left[-i F_{1}\left(\phi_{x}, \phi_{y}\right)\right]\right)= \\
& \delta\left(\phi_{x}-\phi_{y}\right)\left(1-\exp \left[-i F_{1}\left(\phi_{x}, \phi_{y}\right)\right]\right) .
\end{aligned}
$$

Due to (31), the previous expression is clearly vanishing and thus the left hand side of (32) is vanishing too. The Green's function $\Delta_{F}(x, y)$ has therefore the final form

$$
\begin{gathered}
\Delta_{F}(x, y)=\int \frac{d^{4} p}{(2 \pi)^{4}} \frac{1}{p^{2}-m^{2}} \exp \left[-i(p \cdot(x-y))-\frac{i}{2(k \cdot p)} \int_{(k \cdot y)}^{(k \cdot x)} d \phi\left(2 q(A \cdot p)-q^{2} A^{2}\right)\right] \\
T\left\{\operatorname { e x p } \left[\frac{1}{2(k \cdot p)} \int_{(k \cdot y)}^{(k \cdot x)} d \phi\left(q+\frac{e \mu}{2}\right)(\gamma \cdot k)\left(\gamma \cdot A^{\prime}\right)-\right.\right. \\
\left.\left.\frac{e \mu}{2 m}\left((\gamma \cdot k)\left(A^{\prime} \cdot p\right)-q(\gamma \cdot k)\left(A^{\prime} \cdot A\right)-\left(\gamma \cdot A^{\prime}\right)(k \cdot p)\right)\right]\right\}
\end{gathered}
$$

and $S_{F}(x, y)$ can be immediately obtained by (27). The discussion we have done on the special cases $q \neq 0, \mu=0$ and $q=0, \mu \neq 0$ when solving for the wavefunction $\psi(x)$, holds for the Green's function too.

\section{The Hamilton-Jacobi equation}

In this section we want to comment on some aspects of the pseudo-classical nature of the solutions we have determined. The discussion involves in a natural way the properties of the Hamilton-Jacobi equation and the corresponding characteristic functions. We recall that, according to Dirac, the extended Hamiltonian is written as a linear combination of the firstclass constraints $\chi$ and $\chi_{D}$ with arbitrary coefficients, namely

$$
\mathcal{H}=\alpha_{D} \chi_{D}+\alpha_{1} \chi
$$

where $\alpha$ and $\alpha_{1}$ are arbitrary Lagrangian multipliers. In particular, the choice $\alpha_{D}=0$ and $\alpha_{1} \neq 0$ yields

$$
\begin{gathered}
\mathcal{H}=\alpha_{1}\left[(p-q A)^{2}+\frac{i e \mu}{m}\left(p_{\mu}-q A_{\mu}\right) F^{\mu \nu} \xi_{\nu} \xi_{5}-m^{2}+i\left(q+\frac{e \mu}{2}\right) F^{\mu \nu} \xi_{\mu} \xi_{\nu}+\right. \\
\left.\frac{\mu^{2} e^{2}}{16 m^{2}} F^{\mu \nu} F^{\rho \sigma} \xi_{\mu} \xi_{\nu} \xi_{\rho} \xi_{\sigma}\right]
\end{gathered}
$$


and the corresponding covariant Hamilton-Jacobi equation turns out to be

$$
\begin{aligned}
\left(\frac{\partial S}{\partial x^{\mu}}+q A_{\mu}\right)^{2}-\frac{i \mu e}{m}\left(\frac{\partial S}{\partial x^{\mu}}+q A_{\mu}\right)\left(k^{\mu} a^{\nu}-k^{\nu} a^{\mu}\right) \xi_{\nu} \xi_{5} f^{\prime}-m^{2}+ & \\
2 i\left(q+\frac{e \mu}{2}\right)(k \cdot \xi)(a \cdot \xi) f^{\prime} & =0
\end{aligned}
$$

The structure of the Dirac extended Hamiltonian suggests, for the pseudo-classical characteristic function, the factorized form

$$
S=-(p \cdot x)-\frac{1}{2(k \cdot p)} \int_{-\infty}^{(k \cdot x)} d \phi\left(2 q(A \cdot p)-q^{2} A^{2}\right)+S_{D}((k \cdot x))
$$

as a function of the coordinates $x^{\mu}$ and of the initial momentum $p^{\mu}$ and $p^{\mu}$ satisfies the massshell condition $p^{2}=m^{2}$. We therefore obtain for the unknown term $S_{D}((k \cdot x))$ the following first-order differential equation

$$
\begin{gathered}
\frac{d S_{D}}{d \phi}+\frac{i \mu e}{2 m(k \cdot p)}\left[-(k \cdot p)\left(A^{\prime} \cdot \xi\right) \xi_{5}+\left(A^{\prime} \cdot p\right)(k \cdot \xi) \xi_{5}-q\left(A^{\prime} \cdot A\right)(k \cdot \xi) \xi_{5}\right] \\
-\frac{i}{(k \cdot p)}\left(q+\frac{e \mu}{2}\right)(k \cdot \xi)\left(A^{\prime} \cdot \xi\right)=0
\end{gathered}
$$

The complete solution for the characteristic function of the Hamilton-Jacobi equation is then

$$
\begin{array}{r}
S=-(p \cdot x)-\frac{1}{2(k \cdot p)} \int_{-\infty}^{(k \cdot x)} d \phi\left(2 q(A \cdot p)-q^{2} A^{2}\right)+\frac{i}{(k \cdot p)} \int_{-\infty}^{(k \cdot x)} d \phi\left[\left(q+\frac{e \mu}{2}\right)(k \cdot \xi)\left(A^{\prime} \cdot \xi\right)-\right. \\
\left.\frac{e \mu}{2 m}\left(-(k \cdot p)\left(A^{\prime} \cdot \xi\right) \xi_{5}+\left(A^{\prime} \cdot p\right)(k \cdot \xi) \xi_{5}-q\left(A^{\prime} \cdot A\right)(k \cdot \xi) \xi_{5}\right)\right]
\end{array}
$$

Taking into account the realization (7) for the quantized Grassmann variables we finally obtain for the action of $\exp (i \hat{S})$ on the constant spinor $u(p, s)$

$$
\begin{gathered}
\frac{\exp (i \hat{S})}{\left(2 p_{0} V\right)^{1 / 2}} u(p, s)=\frac{1}{\left(2 p_{0} V\right)^{1 / 2}} \exp \left[-i(p \cdot x)-\frac{i}{2(k \cdot p)} \int_{-\infty}^{(k \cdot x)} d \phi\left(2 q(A \cdot p)-q^{2} A^{2}\right)\right] \\
\exp \left[\left(\frac { 1 } { 2 ( k \cdot p ) } \int _ { - \infty } ^ { ( k \cdot x ) } d \phi \left[\left(q+\frac{e \mu}{2}\right)(\gamma \cdot k)\left(\gamma \cdot A^{\prime}\right)-\right.\right.\right. \\
\left.\frac{e \mu}{2 m}\left(-(k \cdot p)\left(\gamma \cdot A^{\prime}\right)+\left(A^{\prime} \cdot p\right)(\gamma \cdot k)-q\left(A^{\prime} \cdot A\right)(\gamma \cdot k)\right)\right] u(p, s)
\end{gathered}
$$

where $\hat{S}$ is the quantized version of $S$.

Contrary to the results previously known in literature for the scalar and Dirac particles [11, 13, 14, we can see that the semi-classical results are not now coincident with the general quantum solutions, but for the two particular cases we have previously discussed. 


\section{References}

[1] F. A. Berezin and M. S. Marinov, Ann. Phys. (N.Y.) 104, 336, (1977).

[2] A. Barducci, R. Casalbuoni and L. Lusanna, Il Nuovo Cimento 35A, 377, (1976).

[3] L. Brink, S. Deser, B. Zumino, P. Di Vecchia and P. Howe, Phys. Lett. B46, 435, (1976);

[4] L. Brink, P. Di Vecchia and P. Howe, Nucl. Phys. B118, 76, (1977)

[5] A. Barducci, R. Casalbuoni and L. Lusanna, Nucl. Phys. B124, 93, (1977)

[6] A. Barducci, R. Casalbuoni and L. Lusanna, Nucl. Phys. B124, 521, (1977)

[7] F. Bordi and R. Casalbuoni, Phys. Lett. B93, 308, (1980); A. Barducci, F. Bordi and R. Casalbuoni, Il Nuovo Cimento B64, 287, (1981).

[8] M. Henneaux and C. Teitelboim, Annals Phys. 143, 127 (1982).

[9] E. S. Fradkin and D. M. Gitman, Phys. Rev. D 44, 3230 (1991).

[10] D. M. Gitman and I. V. Tyutin, Class. Quantum Grav. 7, 2131 (1990).

[11] A. Barducci and R. Giachetti, Il Nuovo Cimento A29, 256, (1975)

[12] N. Boudiaf, T. Boudjedaa and L. Chetouani, Eur.Phys.J. C20, 585, (2001)

[13] D.M. Volkov, Zeits. Phys. 94, 25, (1935); V. Berestetski, E. Lifchitz, L. Pitayevski, Théorie Quantique Rélativiste, Editions MIR (Moscou 1973 ).

[14] A. Barducci and R. Giachetti, J.Phys A 36, 8129, (2003),A. Barducci and R. Giachetti, J.Phys A 38, 1615, (2005)

[15] A. Barducci, Phys. Lett. B118, 112, (1982)

[16] D. M. Gitman and A. V. Saa, Class.Quant.Grav. 10, 1447,(1993).

[17] D. M. Gitman and A. V. Saa, Mod.Phys.Lett. A8, 463,(1993).

[18] N. Boudiaf, T. Boudjedaa and L. Chetouani, Eur.Phys.J. C22, 593, (2001)

[19] J. D. Bjorken and S. D. Drell, Relativistic Quantum Mechanics, McGraw-Hill Book Company (New York 1964)

[20] L. S. Brown and T. W. B. Kibble, Phys. Rev. 133,A705, (1964) 